\title{
LES HISTORIENS DANS L'ESPACE PUBLIC
}

Les débats sur les procès pour crimes contre l'humanité ont associé, sur le mode de l'évidence, l'expertise des historiens à la justice. Il ne s'agit là pourtant que d'un aspect limité au sein d'un phénomène plus ample et plus diversifié. Au delà de la définition ordinaire de l'expertise comme réponse apportée à une question ponctuelle, ce sont les diverses fonctions sociales de l'histoire et la place des historiens dans l'espace public qui doivent être examinées.

\section{UNE DEFINITION LIMINAIRE}

L'expertise historienne, tout en posant des problèmes spécifiques, n'est à nos yeux qu'un cas particulier d'une question qui touche l'ensemble des sciences sociales. On considérera, en première analyse, que relève de l'expertise le discours d'un agent doté d'un savoir ou d'un savoir-faire lorsqu'il vise à répondre à une demande sociale ou à intervenir sur l'organisation sociale. La demande sociale peut être explicite, comme dans le cas d'une commande d'une autorité publique ou d'un groupe secondaire (institution, association, parti politique) (Tanguy, 1995). Elle peut être aussi implicite. L'auteur se constitue alors en porte-parole d'une demande sociale diffuse, et donc susceptible d'être dévoilée (Bourdieu, 1993), voire, à la limite, invente un besoin social (Memmi, 1996). Dans tous les cas, si l'effet de connaissance peut prêter à discussionau motif des règles du métier ou de la nécessité de l'élaboration scientifique des questions posées à la réalité -, cet effet ne peut être purement et simplement occulté ou récusé. Lorsque la distinction de la demande et de la fonction sociale s'efface, c'est à dire lorsque la demande sociale est construite par le savant ou l'expert, la « situation d'expertise » (Cresal, 1995) disparaît aussi au profit d'un projet plus au moins autonome de service à la société ou d'action pratique sur la société. Dire qu'à côté des expertises mandatées existent des expertises autonomes (Castel, 1995), c'est affirmer que le mandat ne constitue pas l'expertise ; c'est aussi faire l'hypothèse que, à s'en tenir dans la délimitation du champ de l'expertise au seul critère de la commande, on aboutit soit à en exclure certains produits ou textes soit à en fausser ou à masquer le sens véritable. Au contraire, élargir la catégorie aux interventions autonomes permet de mettre en lumière le caractère hybride ou délibérément pragmatique d'écrits savants ou de pratiques scientifiquement informées dont la dimension politique était oubliée ou occultée. Ainsi par exemple, le fait de qualifier d'expertise la préface à la seconde édi- 
tion De la division du travail social présente au moins l'intérêt de rappeler la visée pratique du sociologue. Plus fondamentalement, cette perspective, en posant la question des rapports entre le savoir et l'action, ouvre la voie à une réflexion sur les formes de l'engagement et de l'intervention politique et sociale des savants, et globalement des intellectuels.

L'acception de l'expertise qui émerge ainsi récuse l'alternative trop simple entre connaissance et intervention pratique, paradoxalement partagée par les tenants d'une science supposée exempte de tout compromis avec l'air du temps et par ceux qui, à l'inverse, survalorisent l'utilité immédiate des savoirs. Quand les premiers suspectent les seconds de n'être que de médiocres savants aux compétences étroitement spécialisées, les autres symétriquement disqualifient la tentation de la «tour d'ivoire » et, dans le même mouvement, stigmatisent comme "prophètes " quiconque en sort cependant pour prendre position dans l'espace public. L'intellectuel spécifique, tel qu'il a été défini par Michel Foucault, indique, nous y reviendrons, qu'existe au moins une tierce position.

\section{LA FRONTIERE ENTRE CONNAISSANCE ET ACTION}

Nous avons présumé, dans la définition liminaire, qu'un discours d'expertise mobilise des compétences et des savoirs. Gérard Noiriel, dans son dernier livre (1999), applique quant à lui la catégorie à toute œuvre d'apparence "scientifique » qui se contenterait de reprendre les enjeux en débat dans l'espace public sans opérer de rupture avec le sens commun ni, a fortiori, de construction autonome d'objet. Se donnant pour objet les questions sociales chaudes ou les problèmes politiques du jour, ces discours, inscrits dans l'espace public et socialement intéressés, rempliraient nécessairement une fonction sociale ou répondraient à une finalité politique. Pour Gérard Noiriel, ce serait notamment le cas de l'histoire du temps présent. Il est vrai que celle-ci ne «s'intéresse pas simplement à un 'passé mort' mais à un passé proche, porté par la présence d'acteurs et de témoins vivants, et plus encore à ce qui du passé reste encore vivace et actif» (Rousso, 1998). Si l'accent mis par Gérard Noiriel sur ce que nous pourrions encore appeler l'espace public de l'histoire rencontre bien, de fait, un des fondements de notre définition, on ne saurait pour autant souscrire à l'ensemble du propos. En soulignant dans un ouvrage antérieur que «tous les individus qui exercent le métier d'historien » se trouvent engagés dans "l'ensemble des activités de savoir, de mémoire et de pouvoir» qui constituent ce métier, Gérard Noiriel invitait d'ailleurs à une analyse des pratiques historiennes difficilement conciliable avec une caractérisation sommaire de l'expertise et de la science (1996). Car, à bien considérer l'expertise, au delà de ses formes les plus étroites, celle-ci associe en général - et notamment dans le cas de l'expertise historienne - savoir/demande sociale/intervention sociale, production de connaissance et visée pratique assumée. C'est en ce sens que la définition que nous adoptons se rapproche de la notion d'« engagement sociologique » (Mauger, 1995) ou encore de la conception de l'« intellectuel spécifique» de Michel Foucault. La particularité de celle-ci réside en effet dans l'articulation d'une vérité scientifique « locale » et d'un engagement politique, la politisation s'opérant à partir de l'activité scientifique (Foucault, 1994). La catégorie d'expertise proposée par Gérard Noiriel pose problème car elle amalgame des pratiques historiennes différentes. Taxer d'expertise 
tout discours jugé insuffisamment scientifique ou toute recherche s'inscrivant dans l'espace des différends est évidemment une manière de disqualifier une large part de l'histoire politique, notamment des travaux d'histoire contemporaine. Mais où alors tracer la frontière entre histoire chaude et histoire froide ? L'histoire de la Révolution française, centrale dans le « catéchisme révolutionnaire » ou républicain, tomberait sous la critique (Febvre, 1995 ; Furet, 1978), comme d'ailleurs toute histoire qu'une politisation soudaine projette dans l'espace public. À terme, c'est en fait tout le champ de l'histoire politique qui potentiellement devient suspect. À tout prendre, il faudrait alors dénier tout effet de connaissance à l'histoire au motif qu'existent des usages, éventuellement abusifs, de l'histoire (Nietzsche,1988).

Plus généralement, une conception étroite du couple science/expertise aboutit à laisser dans l'ombre les fins et les usages sociaux et politiques des savoirs. Un des dangers d'une disjonction de principe entre science et expertise est de masquer le lien entre la connaissance et l'action, de faire comme si les sciences sociales n'avaient pas été aussi pensées, de Condorcet à Comte, de Durkheim à Mauss, comme fondement de l'agir (Filloux,1977 ; Fournier, 1994). Faut-il souligner encore que la connaissance elle-même est action, ou que l'action peut être un effet escompté de la connaissance, que le dévoilement de la réalité est un effet sur la réalité ? Chez les historiens, Marc Bloch en particulier avait souligné ce lien entre le savoir et l'agir en considérant que la méconnaissance du passé compromet l'action présente. «L'ignorance du passé ne se borne pas à nuire à la connaissance du présent, elle compromet, dans le présent, l'action même. » (Bloch, 1993, 23). Par ailleurs, une stricte définition de la connaissance scientifique aboutit à dresser une frontière trop étanche entre science et valeurs. C'est sans doute une des questions fondamentales posées par ce dossier que, s'il est nécessaire de distinguer textes scientifiques et textes d'intervention, la compréhension aussi bien du travail de la pensée que de ses productions ne peut se satisfaire d'une conception trop rigide, à la limite scientiste, de la frontière entre l'univers de la connaissance et l'univers des fins. S'il est légitime de souligner le nécessaire travail de réélaboration des visions du monde, des projets et des enjeux qui commandent l'intérêt pour tel ou tel objet historique ou social, à force d'insister sur leur nécessaire transmutation, on risque de négliger, dans la genèse de la pensée, l'hybridation qui féconde le travail, et de mésestimer ce que le travail scientifique retient des problématisations politiques ou éthiques (Weber, 1965). Il y a là un équilibre à tenir, qui certes n'est pas facile. Peut-être convient-il, au bout du compte, de souscrire plus modestement au double souci d'échapper à une caractérisation de pure disqualification de l'« expertise » et d'examiner cependant ce qui - du point de vue même de nombre d'historiens du temps présent - pose effectivement problème quand les acteurs du monde social - quels qu'ils soient - formulent des questions à l'adresse voire même, par le jeu des médias, à la place des savants. En d'autres termes, la demande sociale (explicite ou implicite, donnée ou construite), ou l'utilité sociale des savoirs (aveugle à elle-même ou revendiquée comme telle) sont des formes de réalité qu'il ne suffit pas de disqualifier sans autre forme de procès pour atteindre la «scientificité». Plus encore, la position qui se donne pour critique des faux savoirs constitués dans l'air du temps semble oublieuse de ce que «toute histoire est contemporaine». Elle constitue finalement une forme de régression parce que ceux qui la tiennent occultent de fait leurs propres présupposés et négligent notamment que toute histoire, bon gré mal gré, se trouve en quelque 
sorte encadrée, en amont et en aval, par ce qu'il est convenu d'appeler la « mémoire » (Valensi, 1993), par quoi on entend ici les usages de l'histoire.

\section{LES PIECES DU DOSSIER}

Les contributions rassemblées dans ce dossier articulent donc l'expertise - entendue au sens de mobilisation finalisée de savoirs et de compétences - à la demande sociale ou à l'engagement. L'article de Jean Clément Martin montre, à partir de la querelle historiographique sur le « génocide vendéen », comment on serait passé dans la "communauté des historiens » d'un état de «controverse », interne au champ scientifique, à un état de polémique, impliquant l'intervention de différents secteurs, notamment journalistique et politique. Cette situation - somme toute banale au delà de ce cas exemplaire - pose de manière structurelle la question du jugement en Histoire. Et Jean Clément Martin, partie prenante de la querelle qu'il restitue ici, de souligner que l'histoire «n'est pas une activité sans lien étroit avec l'éthique et la morale et qu'elle travaille trop sur le présent pour que son rapport au passé soit désincarné. »' 1 .

Dans «'Destruction Créatrice', transformer le système universitaire est-allemand : le cas de l'histoire ", Konrad Jarausch restitue les mutations de la recherche et de l'enseignement après la réunification. Directeur du Centre d'études d'histoire contemporaine de Postdam, institution créée après la réunification et regroupant des chercheurs de l'Ouest et de l'Est, Konrad Jarausch indique à diverses reprises la place, de l'intérieur, à partir de laquelle il évalue la situation et, eu égard au cadre que celle-ci lui impose, n'esquive cependant aucune question sensible. Dressant un bilan contrasté du processus pour aboutir à un jugement finalement nuancé, il décrit notamment les dispositifs d'expertise scientifique et d'évaluation politique auxquels ont collaboré activement les historiens de l'Ouest. C'est là sans doute, dans l'histoire récente, une situation extraordinaire où des historiens ont eu à juger et à statuer sur l'avenir d'autres hommes qui étaient considérés, dans un autre système social et politique, comme des historiens. Plus fondamentalement, quand cet article rappelle les effets connus et stérilisants de l'esprit de parti et du marxisme-léninisme comme science de l'histoire sur la recherche et l'enseignement, il met également au jour, au delà des bénéfices incontestables de la démocratie recouvrée dans le monde académique, les effets d'une «politisation inversée » et souligne de ce fait le caractère conjoncturel, par essence présent, des interprétations historiques et des choix d'objets.

À cet égard, le texte de Peter Schöttler sur l'histoire dans l'Allemagne nazie offre un exemple paradigmatique sinon caricatural. "De l'histoire rhénane » s'attache à

1. Dans une problématique résolument non positiviste, l’historien J.C. Martin écrit que « l'histoire n'est pas seulement une activité intellectuelle d'observation, sans aucun lien avec l'éthique, ni même avec la morale dans ce qu'elle a de plus étroit dans la fabrication de la mémoire. Par définition, l'Histoire travaille le passé pour répondre aux exigences du présent, et ce faisant elle modèle le présent autant qu'elle recompose les cadres de la mémoire collective... Par ce travail en profondeur, indépendamment des leçons de morale explicites qui peuvent être adjointes à ses démonstrations, elle contribue de manière essentielle à forger des orientations collectives. La présence de ce volet à portée morale et idéologique, dont toute la répercussion est saisissable dans le cours des activités d'enseignement, empêche, à nos yeux, que l'Histoire puisse être rangée sans difficulté dans l'ensemble des Sciences sociales, et fait que la tâche de l'historien trouve là des résonances avec celles du juge. » (Martin, J.C, 1998, 15). 
l'une des écoles historiques dont les travaux ont justifié les revendications nationales des dirigeants nazis. On retrouve là deux problèmes qui sont liés ; le premier renvoie à la frontière entre engagement et « accommodement» (Burrin, 1995, Genèses 1995) ; le second a trait aux valeurs et représentations politiques énoncées sous couvert de science. En l'occurrence, dans cette opération de conversion, se combinent scientisme, tradition du service de l'État, diffusion de l'idéologie volkisch chez les « mandarins », carriérisme et peur (Ringer, 1992 ; Frei, 1994). L'article de Peter Schöttler, version remaniée d'une communication au Congrès des historiens allemands en 1998, constitue en lui-même une intervention de nature politique et s'inscrit à sa manière, encore que sur un front jusqu'ici inexistant, dans les débats sur le national-socialisme qui ne cessent d'agiter la société allemande et dont la fameuse «querelle des historiens » est en France l'épisode le plus connu (Devant l'histoire, 1988).

Le texte de Christian Ingrao «Les historiens et le nazisme. Pratique historiographique, légitimation et engagement », qu'on trouvera à la suite de celui de P. Schöttler, éclaire le contexte de cette intervention. Élargissant le propos, il rend compte des études récentes sur la période et souligne notamment le caractère novateur, d'un point de vue historiographique, des travaux de ces historiens qui ont contribué à la légitimation du nouveau régime.

Jean-Jacques Becker, pour sa part, montre à propos de la question des « responsabilités allemandes $»$ au lendemain de la première guerre mondiale, comment cette question, éminemment politique et patriotique, a donné naissance à la constitution d'une école historique en France. Cette école s'est progressivement détachée de la logique d'expertise - entendons ici tout à la fois réponse à la demande sociale et adhésion, implication personnelle des historiens - qui l'avait engendrée pour passer de la question des responsabilités à celle des origines et finalement des causes, notion qui évacue le jugement et met l'accent sur l'interdépendance des actions.

Dans son article, Dominique Damamme, à partir de L'Étrange défaite de M. Bloch, fait l'hypothèse que le paradigme des Annales n'a pas été sans effets dépolitisants sur l'engagement politique de l'historien et analyse le sens du retour du politique dans ce texte célèbre, appréhendé ici comme un acte d'expertise, c'est à dire un mélange de fait de pratique politique et de pratique scientifique.

Un dossier de cette nature ne pouvait bien sûr éviter la question de Vichy et de ses interprétations, dont les enjeux sensibles ont été à bien des égards à l'origine de notre réflexion. Dans la mesure où tous les historiens français spécialistes de la période nous semblaient ipso facto trop fortement impliqués dans les débats publics, qui constituent pour partie notre objet, nous avons fait appel à un universitaire étranger, américain, compte tenu du rôle joué par Robert Paxton dans l'historiographie de Vichy. Richard Golsan met en lumière ici les raisons de l'intérêt des universitaires américains pour Vichy, en montrant d'ailleurs que cet intérêt renvoie à une sensibilité socialement partagée. À partir de la double vision, savante et non-savante, de la question de Vichy et du fascisme, Richard Golsan élargit son propos, en introduisant différents cas récents d'usage polémique de l'histoire politique américaine et en comparant les positions des historiens aux États-Unis et en France 2.

2. On regrettera l'absence dans ce dossier d'une étude de l'histoire de l'expertise historienne depuis l'affaire Dreyfus. 


\section{LES FORMES DE L'EXPERTISE}

Si nous avons souhaité traiter non de l'expertise entendue au sens le plus étroit du terme mais de la place des historiens dans l'espace public, laquelle inclut la question de l'expertise, celle-ci renvoie à des situations et à des rapports au social et au politique extrêmement divers. À revenir, au delà des cas examinés, à une réflexion plus générale, il nous a semblé possible d'ordonner et de problématiser les modalités variées d'intervention que recouvre cette notion en distinguant trois grandes catégories d'expertise historienne, le conseil, l'expertise judiciaire et l'expertise autoinstituée, sous les deux espèces d'une part de l'histoire judiciaire, d'autre part, pour reprendre une formule de Marc Bloch, de «l'histoire pragmatique » (d'où la formulation adoptée ailleurs d'expertise pragmatique). Par histoire pragmatique, nous entendons une histoire consciente de l'action qu'elle constitue ${ }^{3}$.

\section{LE CONSEIL}

Du conseiller du pouvoir, Ernest Lavisse, « l'instituteur national » (Nora, 1997), constitue sans doute la figure archétypale, même s'il ne fut évidemment pas la première incarnation du genre. Il suffit d'évoquer l'office d'historiographe royal. Ce rappel, moins anecdotique qu'il ne paraît, pose la question plus générale de la contribution des historiens à la fabrication des représentations de l'État et de la nation (Nora, 1997 ; Johnston, 1992 ; Thiesse, 1999). L'autonomisation progressive du champ historique a desserré au $\mathrm{XX}^{\mathrm{e}}$ siècle les liens qui le rattachaient au pouvoir politique, qu'il s'agisse de l'historiographie libérale ou de l'historiographie positiviste - Lavisse, Aulard, Seignobos... - (Dumoulin, 1992 ; Noiriel, 1999). Mais cette évolution ne signifie pas qu'ils aient disparu. Et s'il est vrai que les historiens ne participent plus de manière aussi naïve qu'un Lavisse à la construction et à la légitimation de la nation, il leur arrive d'y contribuer par d'autres voies, volontairement ou non - Cf. Les Lieux de mémoire de Pierre Nora. Sans doute, dans cette perspective, aurait-il fallu ouvrir le chantier de la participation des historiens à l'élaboration des politiques scolaires et des politiques culturelles (Prost, 1983). D'autant plus que si la bibliographie est riche (Nora,1997 ; Prochasson, 1993 ; Ory, 1994 ; Charle, 1994 ; Amalvi,1979 ; Urfalino, 1989 ; Frank, 1995 ; Poulot, 1997 ; Dubois, 1999 ; ...), il n'existe pas de synthèse. Il est vrai qu'il s'agit là d'une question très vaste, surtout si on y associe, comme il est légitime le faire, l'expertise invisible auprès des partis et des associations. L'histoire devenant une marchandise au service des entreprises culturelles de masse, les experts historiens ont investi leur savoir dans les politiques du patrimoine ou les politiques locales (Poirier, 1994 ; Dubois, 1998) : écomusées, musées de pays, musée d'histoire locale, musées des arts et traditions populaires, musées des guerres mondiales... Ils ont été sollicités pour des entreprises de commémoration (Kaplan, 1993) : histoire ou mémoire d'un lieu, d'un groupe, voire d'une entreprise que l'historien consultant légitime en lui faisant don du passé (Bel-

3. À ce titre, la notion, ici mise en œuvre, se distingue à la fois de l'histoire dite «pragmatique » parce que étroitement empirique et de l'histoire "pragmatiste », tel que le modèle en a été proposé notamment par Bernard Lepetit - comme histoire des interactions et des conventions (1995)- et reformulé et appliqué par Gérard Noiriel à une approche des pratiques historiennes elles-mêmes (1996). 
tran et alii, 1995). Par là, on touche à la question peu connue des contrats d'édition et d'études dont le développement constitue une des transformations importantes du métier d'historien (Charle, 1995). Il est évident que les fonctions de conseil ont connu un développement décisif avec l'essor des sciences sociales, dont la figure de savant-expert de Michel Crozier (1964) offre une image évidente. Mais l'appel aux historiens tient aussi à deux autres facteurs liés entre eux, le fait que les historiens français sont depuis le $\mathrm{XIX}^{\mathrm{e}}$ siècle, pour adapter la formule de Joseph Ferrari, des « historiens salariés de l'État» (Johnston, 1992), et d'autre part la place occupée par l'histoire dans la culture et la langue politique en France. Comme l'écrit Antoine Prost, « la société française s'est représentée à elle-même par l'histoire, elle s'est comprise par l'histoire. En ce sens, il est profondément exact que l'histoire fonde l'histoire nationale » (Prost, 1996 ; Joutard, 1993). S'ajoute à ces spécificités le culte très contemporain de la mémoire qu'illustrent aussi bien le succès d'une entreprise éditoriale comme Les lieux de mémoire que le développement des politiques du patrimoine et de la muséification, dans une sorte d'urgence et de passion conservatoire, au risque d'enfouir le présent sous le passé, comme le rappelait Michel de Certeau (de Certeau,1974).

\section{L'EXPERTISE JUDICIAIRE}

Cependant, dans la période récente, c'est une autre forme d'expertise, l'expertise judiciaire, qui suscite débats et controverses. Elle renvoie aux diverses contributions apportées par les historiens à la justice ou à la connaissance d'une violation du Droit. Comme on sait, en France l'appel judiciaire à l'histoire peut être daté de l'affaire Dreyfus (Duclert, 1994). Son développement actuel est étroitement lié aux poursuites intentées à partir des années soixante-dix contre les auteurs de crimes contre l'humanité : inculpation de Jean Leguay en 1979, procès de Klaus Barbie en 1987, inculpation de Bousquet de 1991, de Paul Touvier en 1973 et procès en 1994, procès de Maurice Papon en 1997-1998 (Rousso et Conan,1994). Si le «syndrome de Vichy » (Rousso, 1987) n'explique pas l'invention de la catégorie de "crime imprescriptible » par le législateur en 1964, le recours à cette catégorie, tel qu'il s'est développé ces dernières années, en est bien le symptôme. Il résulte de la conjugaison de la thématique des Droits de l'homme, de l'occultation de la « question juive » à la Libération (Le Monde,1/10/1997, Rousso) et de la découverte par les jeunes générations de ce passé sinon "indicible» du moins longtemps inaudible (Pollak,1990, Wievorka, 1992). C'est dans ce contexte que, parallèlement à la justice, différentes institutions ont fait appel aux historiens : l'archevêque de Lyon a demandé à une commission d'établir les complicités dont Paul Touvier avait pu bénéficier auprès de membres du clergé (Rémond,1992). Une autre commission, à nouveau présidée par René Rémond, a été réunie pour déterminer les conditions dans lesquelles avait été constitué et conservé le «fichier juif» (1996). Des commissions ont été crées pour établir les responsabilités de différentes institutions pendant la guerre dans la déportation des juifs ou la spoliation des biens juifs (commission Mattéoli sur l'aryanisation en France, commissions sur la ville de Paris, l'INSEE, la SACEM, la CDC, les musées nationaux, les banques). À chaque fois, des spécialistes ont participé aux travaux de ces commissions pour établir les faits ou pour déterminer les responsabilités. 
On trouve dans Un passé qui ne passe pas d'Henry Rousso et d'Eric Conan ou encore dans La Hantise du passé (Rousso,1998) une critique aiguë de la judiciarisation de l'histoire. Au delà de mises en garde sur la valeur «pédagogique » de ce type de procès ou d'interrogations, sur l'effet de clôture symbolique et pratique de ces procès, la critique est double : elle vise la logique d'un questionnement judiciaire imposé à l'historien (dans le cas du procès Papon, l'absence de questions sur le rôle de l'occupant allemand ou sur ce que pouvait être un acte de résistance chez un haut fonctionnaire a été relevé) et, deuxièmement, le façonnage de l'histoire, l'opération judiciaire de qualification travestissant les faits pour les faire entrer dans le cadre de la définition jurisprudentielle du crime contre l'humanité, comme ce fut le cas lors du procès Touvier ${ }^{4}$. Au nom de ce qu'il est convenu d'appeler le « devoir de mémoire », les historiens ont pour la plupart accepté de témoigner à Bordeaux et ont considéré respecter le double impératif de justice et de vérité en déposant sur le «contexte» dans lequel avait agi Maurice Papon. En validant le rôle de témoin chargé d'éclairer le jury, rôle qui leur était assigné par la justice, les historiens ont consenti, selon Henry Rousso, à un renoncement au savoir historique, et, du même coup, à une instrumentalisation de l'histoire et à une confusion des genres de 1'histoire et de la mémoire ${ }^{5}$. D'où la conclusion, qu' ' en acceptant de jouer le rôle de 'témoin-expert', ils (les historiens) couraient (donc) le risque d'engendrer une confusion entre leur travail scientifique et celui de la cour, entre leurs interprétations et leurs propres jugements de valeur, et ceux de la justice qui devait délivrer une sentence sur des faits précis, imputables à un individu singulier. » (Rousso, 2000, a). Dans les débats autour de l'expertise judiciaire, on a beaucoup dit que l'intervention historienne ne pouvait absolument pas être assimilée à un acte d'expertise, qu'elle n'était en rien comparable à la critique des sources que les chartistes ou d'autres historiens comme Jaurès avaient effectuée au moment de l'affaire Dreyfus, et qu'en somme, comme le suggère François Hartog, la conjoncture historiographique actuelle serait mieux caractérisée par la figure du témoin que par celle de l'expert (Hartog, 1998). Mais c'est évacuer un peu vite le débat. Car ce qui est visé sous la qualification d'expert, ce sont deux choses, d'une part la mise au service de la justice d'une compétence historienne mais aussi la participation des historiens à la formation de la conviction du tribunal, et par là au jugement. Les historiens se défendent de mélanger les genres. Ils disent chercher à expliquer, non à prendre position : l'historien qui « fournit le contexte » ne juge pas, quand bien même assume-t-il une fonction civique ou politique. Selon René Rémond, «c'est le rôle de l'historien,

4. Sur les rapports de l'histoire et du droit, voir Yan Thomas : «...historiens et juristes ne s'appuient pas sur une même idée de la vérité...La factualité même du fait n'est pas de même nature pour les historiens et pour les juristes. Pour les historiens, elle s'impose d'emblée. Pour les juristes, elle est subordonnée à une signification normative antérieure. Avant d'être avérés, les faits sont qualifiés, qualifiés donc pré-jugés... En réalité, ce ne sont pas tant les méthodes qui sont distinctes que l'objet sur lequel elles portent - que le fait même de la vérité dont il s'agit. Pour les uns, le fait n'existe que comme condition d'un jugement de valeur car il n'y a de pertinent à l'évoquer que s'il est nommable juridiquement. Pour les autres, il est englobant, le jugement de valeur lui-même apparaissant comme une donnée... le fait... en droit... est nécessairement prédéterminé », Thomas, 1988, 22-23.

5. De surcroît, dans le procès Papon, les historiens connaissaient moins bien le dossier (et ne pouvaient, aux termes de la procédure d'assises, le connaître) que les juges, sauf pour l'un d'entre eux, Michel Bergès, le seul à s'être prononcé sur la culpabilité de l'accusé mais aussi le seul qui ait cru nécessaire de nuancer considérablement ses premières accusations (Le Monde, 22/10/1997). 
lorsque l'opinion est troublée, que de faire la vérité sans interférer avec l'œuvre de justice » (Rousso et Conan, 1996, 247). La ligne de défense de René Rémond est partagée par l'ensemble des historiens qui participent aux enquêtes ou aux procès, et ce n'est qu'après coup que Robert Paxton a reconnu que la frontière entre l'explication et le jugement n'était pas aussi étanche qu'il le prétendait (Rousso, 2000, a). Pour l'instant, il ne semble pas que les historiens aient répondu aux questions de Yan Thomas. Elles méritent de ce fait d'être citées un peu longuement : «Que se passe-t-il lorsque l'acte (par exemple, avoir accepté de servir d'intermédiaire, par sa signature, entre les ordres criminels d'un préfet et leur exécution par la police) ne prend sa signification juridique, au regard du crime contre l'humanité, que dans la mesure où il s'inscrit dans nombre de circonstances proches et lointaines - déportations... persécutions raciales... L'historien est-il si éloigné du juge, lorsque le dessin qu'il trace du 'contexte' des actions qu'il s'interdit de juger sert précisément à qualifier ces actions comme crimes ?... le crime contre l'humanité a ceci de particulier que cette inclusion du contexte dans l'acte est essentiel à sa définition juridique... Le contexte ici entre dans le définition du crime, l'historien dans la détermination du contexte, la responsabilité collective dans la responsabilité individuelle, le métier d'historien dans l'office du juge... Il n'est vrai de dire que l'historien 'fournit le contexte' que parce qu'il revient au juge d'en investir l'accusé » (Thomas,1998). En une formule ramassée, on pourrait dire, si l'on suit Y. Thomas, que « le contexte» constitue le crime puisque, faute de condamner un État «absolu mais innocent», une histoire collective est incorporée dans un individu. Dans le cas des crimes contre l'humanité, l'historien participerait alors, bon gré mal gré, au jugement.

Si les historiens peuvent dans leur fonction d'expert judiciaire placer, malgré tout, leur contribution sous l'égide de Marc Bloch et de Lucien Febvre, c'est que les fondateurs des Annales, tout en sachant que l'histoire, à la différence du jugement judiciaire, produit moins des preuves que des constructions intelligibles et des enchaînements justifiables, tenaient la sentence pour une différence essentielle entre le juge et l'historien : " Il existe deux formes d'être impartial : celle du savant et celle du juge. Elles ont une racine commune, qui est l'honnête soumission à la vérité : le savant enregistre, bien mieux, il provoque l'expérience qui, peut-être, renversera sa plus chère théorie. Quel que soit le vœu secret de son cœur, le bon juge interroge les témoins sans autre souci que de connaître les faits, tels qu'ils furent. Cela est, des deux côtés, une obligation de compétence qui ne se discute point. Un moment vient, cependant, où les chemins se séparent. Quand le savant a observé et expliqué, sa tâche est finie. Au juge, il reste encore à rendre sa sentence... (Car le savant) ne saurait condamner ou absoudre sans prendre parti pour une table des valeurs, qui ne relève plus d'aucune science positive... On oublie qu'un jugement de valeur n'a de raison d'être que comme la préparation d'un acte et de sens seulement par rapport à un système de références morales, délibérément accepté. »(Bloch, 1993 ; Febvre, 1995). S'ils pouvaient, non sans hésitation d'ailleurs ${ }^{6}$, se référer à cette opposition simple,

6. Dumoulin, (2000, 282 et suivantes). À consulter également Carlo Ginzburg : « Réduire l'historien au juge, c'est simplifier et appauvrir la connaissance historique ; mais réduire le juge à l'historien, c'est pervertir irrévocablement l'exercice de la justice » $(1997,118)$. C. Ginzburg rappelle par ailleurs que «l'histoire se constitue en tant qu'activité intellectuelle... au croisement de la médecine et de la rhétorique. Elle examine cas et situations, en recherchant leurs causes naturelles selon 
c'est qu'ils n'avaient pas été confrontés au problème de la preuve tel que l'a posée depuis les années soixante la question du relativisme, du révisionnisme et a fortiori du négationnisme. C'est le retour du politique et avec lui de l'événement, retour dont, selon P. Nora, il n'y aurait peut être pas lieu de s'étonner au regard des expériences du $\mathrm{XX}^{\mathrm{e}}$ siècle, qui a relancé la réflexion sur le positivisme en histoire. Le paradigme antipositiviste des Annales s'était déjà affronté aux catégories de vérité et de preuve, mais ce sont les ouvrages de Paul Veyne, de Michel de Certeau et de Paul Ricœur qui ont le plus fortement souligné le caractère de construction des opérations historiques ou la nature fictionnelle de l'œuvre historique, sans toutefois que ces thèses prennent le tour radical qu'elles ont connu aux États-Unis (Ricœur, 1991; Chartier, 1998). Or, ce perspectivisme méthodologique s'est heurté dans les années quatre-vingt au regain de l'histoire politique, tout se passant comme si le combat contre les « assassins de la mémoire » (Vidal-Naquet,1987) avait imposé à l'historiographie la question de la preuve (Brayard, 1998). Le débat sur le fait et la preuve, un temps laissé de côté, est donc revenu en force dans l'historiographie (Chartier, 1998), avec une acuité d'autant plus grande qu'il est porté par la tension entre deux formes d'histoire, d'un côté une histoire des pratiques et des représentations, de l'autre une histoire politique, avec des individus singuliers et des moments singuliers. Le retour du politique a engendré ce paradoxe à plusieurs facettes d'une inscription de la question de la vérité au cœur d'un paradigme relativiste dominant (Ginzburg, 1997) ${ }^{7}$.

À cet égard la question de la mémoire, telle qu'elle s'est développée en France depuis le milieu des années soixante-dix, est éclairante. Définie à l'origine moins par ses contenus (les souvenirs, les commémorations, les récits du passé telles que les États, partis, Églises, associations et minorités les élaborent...) que par l'utilisation «stratégique» que les historiens pouvaient en faire pour le «renouveau de l'historiographie » (Nora, 1978), la mémoire, constituée en objet d'une histoire « critique », et à ce titre, effet de la prise en considération par les historiens de la relativité de la connaissance en histoire, est devenue vingt ans plus tard, par un curieux retournement, le fondement d'une exigence de « vérité » et de savoir étroitement positiviste sur le passé. Tout se passe comme si les historiens, dans un contexte marqué par une réflexion d'ordre épistémologique sur les limites du savoir historique, par une sensibilité nouvelle, post-68, pour les acteurs anonymes et dominés de l'histoire et par l'arrivée d'une génération d'historiens nés après la guerre, avaient en quelque sorte contribué à faire naître une demande sociale, qui, bientôt, au nom de la «mémoire », se retournerait directement vers eux.

\section{L'EXPERTISE AUTO-INSTITUEE}

Dernière forme d'expertise, l'expertise auto-instituée. Elle revêt essentiellement deux modalités, celle de l'histoire « judiciaire» et celle de l'« expertise pragmatique ». La première de ces modalités que, par analogie avec l'expertise judiciaire, on nomme histoire « judiciaire », se rapporte à des affaires dans lesquelles la justice a

l'exemple de la première, et les expose en suivant les règles de la seconde- un art de la persuasion né devant les tribunaux » (id, 16).

7. «Les notions de preuve et de vérité sont... partie intégrante du métier d'historien », Ginzburg, 1997, p. 23 
été saisie ou aurait pu l'être. Il y a en France des précédents célèbres, l'affaire Calas, l'affaire Dreyfus, avec Une erreur judiciaire de Bernard Lazare ou Les preuves de Jean Jaurès (Duclert, 1998 a, 1998 b, 1999). Les « enquêtes » sur les crimes de guerre commis par l'armée allemande lors de la première guerre mondiale évoquées dans ce dossier par Jean Jacques Becker, ou plus près de nous, les ouvrages de Serge Klarsfeld (1983, 1985), peuvent également servir d'illustrations. C'est ce type de démarche qui sous-tend L'affaire Audin de Pierre Vidal-Naquet, où l'historien reprend en pleine connaissance de cause le modèle de l'affaire Dreyfus pour démontrer la culpabilité de militaires français dans l'assassinat d'un militant communiste algérois. Le texte le plus récent dans ce genre est celui de Carlo Ginzburg, Le juge et l'historien, où l'auteur réexamine les éléments du dossier d'A. Sofri, militant de l'extrême gauche italienne condamné à perpétuité pour le meurtre d'un commissaire de police sur la base de la déposition tardive et peu crédible d'un repenti. Pratiquée par des historiens de métier, cette forme d'histoire l'est également par des «outsiders », militants d'une cause comme Jean-Luc Einaudi à propos de la manifestation du 17 octobre 1961 à Paris (Einaudi, 1991).

Proches de ces formes, bien qu'ils ne relèvent de l'expertise auto-instituée stricto sensu, l'actualité récente offre plusieurs exemples où des historiens ont été appelés à rendre un avis à la suite de mises en cause ou d'insinuations concernant le passé de personnages publics : la table-ronde qui a réuni autour de Raymond et Lucie Aubrac - et, il convient de le souligner, à leur demande - des historiens spécialistes de la France de Vichy (Libération, 10/11/12-13/08/1997), ou le comité d'experts/jury d'honneur formé à la suite de l'accusation lancée contre Pierre Cot de collaboration avec les services secrets soviétiques (Le Monde, 25/1/1995). Pour le coup, la différence entre enquête historienne et enquête policière s'estompe.

Enfin, la seconde modalité, l'histoire ou l'expertise pragmatique renvoie à des textes d'intervention dans lesquels s'énonce une vision ou un projet politique, sous la forme de jugements, de dispositifs, de programmes, et orientés par suite vers l'espace public. Ces textes, pour lesquels la préface programmatique de Durkheim à La division du travail social a servi de modèle, allient, dans des proportions variables, discours analytique et programmatique. Textes hybrides, ils associent démarche normative et démarche rationnelle, connaissance et jugement. De ce fait, ils ne sont pas coupés de toute règle de méthode scientifique. Qualifier L'Étrange défaite de texte d'intervention ne doit pas empêcher de reconnaître que les analyses qu'il contient sur la société française reposent sur une longue pratique scientifique et que la dénonciation des responsabilités prend progressivement la forme d'une recherche des causes diverses de l'affaissement « moral » de la société française.

Cependant, rapprochement ne signifie pas assimilation. Deux critères permettent de distinguer en droit sinon en fait histoire scientifique et expertise : le registre politique et la dimension programmatique. Il faut d'abord remarquer que l'expertise pragmatique est un genre plus rare en histoire qu'en sociologie ${ }^{8}$. Mais, en même temps, comme on sait, l'histoire a toujours été une propédeutique à l'action. Car elle fut d'abord explicitement une science pratique : Historia magistra vitae, la formule de Ciceron est restée (Koselleck, 1990, 1997). Par la suite, l'Histoire, tout à la fois

8. Voir M. Mauss (1927), C. Levi-Strauss, (1958, 1983), P. Bourdieu. 
concept et processus, a été érigée en tribunal du monde par les philosophies de 1'histoire: "Après que 1' 'histoire' (Geschichte) est devenue un concept réflexif, servant d'intermédiaire entre le futur et le passé et étant capable d'expliquer, de justifier ou de légitimer, sa mission peut être perçue de différentes manières. Les nations, les classes, les partis, les sectes ou tout autres groupes d'intérêts peuvent, doivent même se référer à l'histoire pour autant que la généalogie de leur propre position leur confère des arguments juridiques dans le champ d'action politique ou social » (Koselleck, 1997, 70). Cette histoire, qui se caractérise autant par l'érudition que par la finalité, et qu'on retrouve dans toutes les écoles historiographiques du $\mathrm{XIX}^{\mathrm{e}}$ siècle, libérale (Guizot), conservatrice (Taine, Cochin), républicaine (Michelet, Aulard, Seignobos) ou progressiste (Jaurès), rend incertaine la coupure proclamée et idéalisée entre la science et la politique (Nora, 1978). On voit combien la frontière peut être fluide entre une histoire savante, et tenue pour telle, et une histoire pragmatique, d'autant que l'une et l'autre peuvent viser les mêmes objectifs. Les expertises pragmatiques s'adossent fréquemment à des textes savants ${ }^{9}$, s'appuient sur des règles du métier, voire constituent un savoir historique. Mais entre une Histoire savante, voire même une " histoire critique » (Nora, 1997), et un texte d'intervention subsiste la différence qui tient au passage de l'implicite à l'explicite, de l'énonciation à la dénonciation, du dire au faire, de la sphère scientifique à la sphère politique.

Peut-on repérer des séquences historiques ou des conjonctures qui permettraient de comprendre la rareté ou l'essor de ces divers genres d'expertise ? Cette question, nous n'avons fait que l'effleurer. Certes, la présence du politique réunit ces différentes formes d'intervention, mais chacune d'elles a son histoire propre. Le développement récent de l'expertise au sens du conseil ou de l'aide à la décision (Habermas, 1973) tient à des causes internes à la profession d'historien (sa croissance morphologique notamment), au développement des sciences sociales (Charle,1994; Topalov, 1999), ainsi qu'à des évolutions culturelles plus globales, dont le rapport que la société entretient avec son passé et son identité, ce qu'on appelle la «mémoire ». À ces circonstances, s'ajoutent dans le cas de l'expertise judirique le concours des historiens à une revendication sociale multiforme de justice qui demande à l'État réparation pour le passé ou pour le présent (le sang contaminé), et leur participation, bene volens, au processus de judiciarisation de la politique qui en a résulté. S'agissant de l'expertise pragmatique enfin, il faudrait souligner le rôle des crises politiques (Ernest Renan après la défaite de 1870 ou de Marc Bloch après celle de 1940), des phases de refondation d'un régime (Émile Durkheim), ou plus simplement des alternances politiques comme après 1981. Ces conjonctures ouvrent la possibilité d'une expertise auto-instituée auprès du pouvoir politique.

Ces quelques remarques invitent à une analyse des intersections entre le champ scientifique et le champ politique. On retiendra de ce dossier et des premières réflexions qu'il constitue l'intérêt qu'il y aurait à préciser, à coté des figures polaires de l'expert mandaté ou de l'intellectuel prophétique, les formes médianes d'inter-

9. Pour Durkheim, la science sociale ordonnait l'art social, et pour Bloch, la science historique pouvait être utile à l'action. Les travaux scientifiques peuvent déboucher sur des programmes d'action. D'où l'idée que nous suggérons de savoirs «à effet programmatique », désignant sous ce terme les œuvres dont les énoncés ont la propriété d'étayer des programmes d'action. 
vention des sciences sociales - textes scientifiques ouvrant à des usages pragmatiques, textes plus explicitement politiques portant l'empreinte du savoir scientifique.

Dominique Damamme, Paris IX-Iresco-CSU

Marie-Claire LAVABRE CEVIPOF 


\section{REFERENCES BIBLIOGRAPHIQUES}

Amalvi C. 1979, Les héros de l'histoire de France, Phot'œil.

BEDARIDA F. 1995, L'histoire et le métier d'historien en France 1945-1995, Éditions de la Maison des sciences de l'homme.

BELTRAN et alii 1995, L'histoire d'entreprise en France, essai bibliographique, Cahiers de l'IHTP, $\mathrm{n}^{\circ} 30$.

BLOCH M. 1993, Apologie pour l'histoire ou métier d'historien, Armand Colin, p. 157.

Bourdieu P. 1993, La Misère du Monde, Éditions du Seuil.

Boutier J., Julia D. 1995, Passés recomposés, Autrement.

BRAYARD F. 1998, De la falsification négationniste, ce que mentir implique, 8-10 novembre 1998, Bruxelles, dir. F. BRAYARD et J. KOTEK, La négation de la Shoa, un phénomène de falsification historique. Histoire et mise en perspectives.

BURRIN PH. 1995, La France à l'heure allemande 1940-1944, Éditions du Seuil.

CASTEL R. 1985, L'expert mandaté et l'expert instituant, in Situations d'expertise et socialisation des savoirs, C.R.E.S.A.L.

CERTEAU M. de. 1994, La beauté du mort in La culture au pluriel, UGE.

CERTEAU M. de. 1975, L'écriture de l'histoire, Gallimard.

CHARLE C. 1974, La République des universitaires, Seuil.

CHARle C. 1995, Être historien en France :une nouvelle profession? in BEDARIDA F. 1995, L'histoire et le métier d'historien en France 1945-1995, Éditions de la Maison des sciences de l'homme.

CHARTIER R. 1998, Au bord de la falaise : l'histoire entre certitudes et inquiétudes, Albin Michel.

COLlECTIF, 1988, Devant l'histoire. Les documents de la controverse sur la singularité de l'extermination des Juifs par le régime nazi, Cerf.

CONAN E., Rousso H. 1994 1996, Vichy, un passé qui ne passe pas, Fayard.

COURTOIS S. et alii. 1997, Le livre noir du communisme, Laffont.

COURTOIS S. 1995, Le Monde, 26/12/1995.

CrozIER M. 1964, The Cultural Revolution : Notes on the Changes in the Intellectual Climate in France, Daedalus.

Descombes V. 1991, Sciences sociales, sens social, Critique, juin-juillet 1991.

Dubois V., dir, 1998, Politiques locales et enjeux culturels Les clochers d'une querelle $X I X^{e}-X X^{e}$ siècle, La Documentation française.

DuboIS V. 1999, La politique culturelle. Genèse d'une catégorie d'intervention publique, Belin.

DUCLERT V. 1994, L'affaire Dreyfus, Éditions La Découverte.

DUCLERT V. 1998, Histoire, historiographie et historiens de l'affaire Dreyfus (18941997), in M. LEYMARIE, La postérité de l'affaire Dreyfus, Presses Universitaires du Septentrion.

DUCLERT V. 1998, La Ligue de "l'époque héroïque » : la politique des savants, Le Mouvement Social, ${ }^{\circ} 183$, avril-juin 1998.

DUCLERT V. 1999, L'engagement scientifique et l'intellectuel démocratique. Le sens de l'affaire Dreyfus, Politix, $\mathrm{n}^{\circ} 48$.

DuMOULIN O. 1992, Histoire et historiens de droite, in SiRINELli J.F. dir., Histoire des Droites en France, Gallimard, Tome 2.

EINAUDI J.L. 1991, La bataille de Paris, 17 0ctobre 1961, Seuil. 
FEBVRE L. 1995, Contre les juges suppléants de la vallée de Josaphat, in Combats pour l'histoire, Agora, Armand Colin.

FILlOUX J-C. 1977, Durkheim et le socialisme, Droz.

FOURNIER M. 1994, Marcel Mauss, Fayard.

FrEI N. 1994, L'État hitlérien et la société allemande, Seuil.

FRISCH P. 1985, Situations d'expertise et expert-système, in Situations d'expertise et socialisation des savoirs, C.R.E.S.A.L.

FouCAult M. 1994 [1976], La fonction politique des intellectuels, Dits et écrits 1954-1988, Vol. 3, Gallimard.

FURET F. 1978, Penser la Révolution, Gallimard.

FRANK R. 1995, La place des deux guerres mondiales dans les manuels scolaires, Nouvelle Alternative, $\mathrm{n}^{\circ} 37$.

GENESES 1995, Le nazisme et les savants, $\mathrm{n}^{\circ} 21$.

GINZBURG C. 1989, Mythes, emblèmes, traces, morphologie et histoire, Flammarion.

GINZBURG C. 1983, Enquête sur Piero della Francesca, Flammarion.

GinZBURG C. 1997, Le juge et l'historien, Considérations en marge du procès Sofri, Verdier, p.118.

GINZBURG C, REVEL J, Table ronde sur « Le juge et l'historien » EHESS, novembre 1997.

HABERMAS J. 1973, La technique et la science comme idéologie, Gallimard.

HARTOG F. 1998, L’historien et la conjoncture historiographique, Le Débat, n 102.

JASPERS K.,1990, La culpabilité allemande, Les Éditions de Minuit.

JEANNENEY J.N. 1998, Le passé dans le prétoire, L'historien, le juge et le journaliste, Le Seuil.

Johnston W. 1992, Post modernisme et bimillénaire Le culte des anniversaires dans la culture contemporaine, PUF.

JOUTARD PH. 1993, Une passion française : l'histoire, in BURGUIERE A., REVEL J., Histoire de France. Les formes de la culture, Paris, Le Seuil.

KAPLAN S. 1993, Adieu 89, Fayard.

KLARSFELD S. 1983 1985, Vichy-Auschwitz. Le rôle de Vichy dans la Solution finale, Fayard.

KNIBIEHLER Y. 1973, Naissances des sciences humaines : Mignet et l'histoire philosophique au XIX $X^{e}$ siècle, Flammarion.

KOSELLECK R. 1990, Le futur passé, E.H.E.S.S.

KOSELLECK R. 1997, L'expérience de l'histoire, Gallimard, Le Seuil.

LE Goff J. 1993, Préface à Apologie pour l'histoire ou Métier d'historien, Armand Colin.

LEPETIT B., L’histoire prend-elle les acteurs au sérieux ?, Espaces-Temps, n59-61.

LEPETIT B. 1995, Les formes de l'expérience, Une autre histoire sociale, Albin Michel.

LeVI-STRAuSS C. 1958, Race et Histoire, Plon.

LEVI-STRAuSS C. 1983, Le regard éloigné, Plon.

MARTIN J.C. 1998, La démarche historique face à la vérité judiciaire. Juges et historiens, Droit et Société, ${ }^{\circ} 38$.

MAUGER G. 1995, L’engagement sociologique, Critique, $\mathrm{n}^{\circ}$ 579-580.

MAUSS M. 1971, Divisions et proportions des divisions de la sociologie, L'Année Sociologique 1927, in Essais de Sociologie, Éditions du Seuil. 
Memmi D. 1996, Les gardiens du corps. Dix ans de magistère bioéthique, Éditions de l'EHESS

NiETZSCHE F. 1988, Seconde considération intempestive De l'utilité et de l'inconvénient des études historiques pour la vie, Flammarion.

NOIRIEL G. 1996, Sur la «crise » de l'histoire, Belin.

NOIRIEL G. 1999, Les origines républicaines de Vichy, Hachette.

NorA P. 1978, Mémoire collective, in J. Le GofF et alii, La Nouvelle Histoire, Retz.

NORA P. 1997, Lavisse, instituteur national, La République, Quarto, Vol. 1, Gallimard.

NoRA P. 1997, «L'Histoire de France» de Lavisse in La Nation, Quarto, Vol. 1, Gallimard.

NORA P. 1995, Le syndrome, son passé, son avenir, in «Forum; the Vichy Syndrome », French Historical Studies, ${ }^{\circ} 2$.

ORY P. 1994, La belle illusion, culture et politique sous le signe du Front populaire, 1935-1938, Plon.

PEAN P. 1995, Une jeunesse française François Mitterrand 1943-1947, Fayard.

POIRIER P. et alii 1994, Jalons pour l'histoire des politiques cultuelles locales, La Documentation française.

POLLACK M. 1994, L'univers concentrationnaire, Métaillé.

Poulot D. 1997, Musée, Nation, Patrimoine, 1789-1815, Gallimard.

Prochasson C. 1993, Les intellectuels, le socialisme et la guerre 1900-1938, Seuil.

Prochasson C., RaSMUSSEN A. 1996, Au nom de la patrie les intellectuels et la première guerre mondiale (1910-1919, La Découverte.

Prost A. 1983, Les lycées et leurs études au seuil du XXI siècle, Ministère de l'Éducation Nationale.

Prost A. 1996, Douze leçons sur l'histoire, Seuil, p. 25.

REMOND R. et alii, 1992, Paul Touvier et l'Eglise, Fayard.

Ricoeur P. 1991, Temps et récit, Éditions du Seuil.

RINGER K. 1992, The decline of German Mandarins, Harvard U.P.

Rousso H. 1987, Le syndrome de Vichy de 1944 à nos jours, Éditions du Seuil.

Rousso H. 1998, La hantise du passé, Textuel.

Rousso H. 2000 (a), Justice, histoire et mémoire en France, Réflexions sur le procès Papon, in D.van LAAK N. FreI, Geschichte vor Gericht. Historiker, Richter und die Suche nach Gerechtigkeit, Verlag C.H Beck.

Rousso H. 2000 (b), Juger le passé ? Justice et Histoire en France, le génocide des juifs entre procès et histoire, in F. BRAYARD ET G. SMITH, Complexe

RAPPORT DE LA COMMISSION D'HISTORIENS CONSTITUEE POUR EXAMINER LA NATURE Des Relations De P. Cot AVec les autorites sovietiques, Le Monde, 25/1/1995.

TANGUY L. 1995, Le sociologue et l'expert: une analyse de cas, Sociologie du Travail, $\mathrm{n}^{\circ} 3$.

THIESSE A-M. 1999, La création des identités nationales, Le Seuil.

THOMAS Y. 1998, La vérité, le temps, le juge et l'historien, Le Débat, ${ }^{\circ} 102$.

Topalov C., (dir). 1999, Laboratoires du nouveau siècle la nébuleuse réformatrice et ses réseaux en France, 1880-1914. Éditions de l'EHESS.

URFALINO P. 1989, Les politiques culturelles : mécénat caché et académies invisibles, L'Année Sociologique, vol. 39.

VALENSI L. 1992, Fables de la mémoire La glorieuse bataille des trois rois, Le Seuil. 
VIDAL- NAQUET P. 1987, Les assassins de la mémoire « un Eichmann de papier » et autres essais sur le révisionnisme, La Découverte.

VIDAL-NAQUET P. 1993, Le trait empoisonné. Réflexions sur l'affaire Jean Moulin, La Découverte.

WEBER M. 1965, Essais sur la théorie de la science, Plon.

WIEVORKA A. 1992, Déportation et génocide entre la mémoire et l'oubli, Plon.

WOLTON T. 1993, Le grand recrutement, Grasset. 